\title{
Correction: SP1-activated long noncoding RNA IncRNA GCMA functions as a competing endogenous RNA to promote tumor metastasis by sponging miR-124 and miR-34a in gastric cancer
}

Yaru Tian · Ranran Ma - Yujing Sun · Haiting Liu - Hui Zhang · Yiyuan Sun · Lei Liu · Yuhong Li · Lin Song · Peng Gao (1)

Published online: 10 September 2020

(c) The Author(s), under exclusive licence to Springer Nature Limited 2020

Correction to: Oncogene

https://doi.org/10.1038/s41388-020-1330-4

The original version of this article contained an error in

Fig. 5. In Fig. 5y, there was a positive correlation between the expression of IncRNA GCMA and snail IHC score and the Spearman $r$ value should be 0.725 instead of -0.725 .

This has now been corrected in both the PDF and HTML versions of the article. 